\title{
TBC1D24 regulates axonal outgrowth and membrane trafficking at the growth cone in rodent and human neurons
}

\author{
Davide Aprile ${ }^{1} \cdot$ Floriana Fruscione $^{2} \cdot$ Simona Baldassari $^{2} \cdot$ Manuela Fadda $^{1} \cdot$ Daniele Ferrante $^{1} \cdot$ Antonio Falace $^{3}$. \\ Emmanuelle Buhler ${ }^{4}$ Jacopo Sartorelli ${ }^{2}$. Alfonso Represa ${ }^{4}$ - Pietro Baldelli $\mathbb{D}^{1,5}$ • Fabio Benfenati $\mathbb{D}^{5,6}$. \\ Federico Zara ${ }^{2} \cdot$ Anna Fassio ${ }^{1,5}$
}

Received: 9 April 2018 / Revised: 25 January 2019 / Accepted: 20 February 2019 / Published online: 11 March 2019

(c) ADMC Associazione Differenziamento e Morte Cellulare 2019

\begin{abstract}
Mutations in TBC1D24 are described in patients with a spectrum of neurological diseases, including mild and severe epilepsies and complex syndromic phenotypes such as Deafness, Onycodystrophy, Osteodystrophy, Mental Retardation and Seizure (DOORS) syndrome. The product of TBCID24 is a multifunctional protein involved in neuronal development, regulation of synaptic vesicle trafficking, and protection from oxidative stress. Although pathogenic mutations in TBC1D24 span the entire coding sequence, no clear genotype/phenotype correlations have emerged. However most patients bearing predicted loss of function mutations exhibit a severe neurodevelopmental disorder. Aim of the study is to investigate the impact of TBC1D24 knockdown during the first stages of neuronal differentiation when axonal specification and outgrowth take place. In rat cortical primary neurons silenced for TBC1D24, we found defects in axonal specification, the maturation of axonal initial segment and action potential firing. The axonal phenotype was accompanied by an impairment of endocytosis at the growth cone and an altered activation of the TBC1D24 molecular partner ADP ribosylation factor 6. Accordingly, acute knockdown of TBC1D24 in cerebrocortical neurons in vivo analogously impairs callosal projections. The axonal defect was also investigated in human induced pluripotent stem cell-derived neurons from patients carrying TBCID24 mutations. Reprogrammed neurons from a patient with severe developmental encephalopathy show significant axon formation defect that were absent from reprogrammed neurons of a patient with mild early onset epilepsy. Our data reveal that alterations of membrane trafficking at the growth cone induced by TBC1D24 loss of function cause axonal and excitability defects. The axonal phenotype correlates with the disease severity and highlight an important role for TBC1D24 in connectivity during brain development.
\end{abstract}

Edited by J. M. Hardwick

Supplementary information The online version of this article (https:// doi.org/10.1038/s41418-019-0313-x) contains supplementary material, which is available to authorized users.

Anna Fassio

afassio@unige.it

1 Department of Experimental Medicine, University of Genoa, Genoa, Italy

2 Laboratory of Neurogenetics and Neuroscience, IRCCS Istituto Giannina Gaslini, Genoa, Italy

3 Pediatric Neurology, Neurogenetics and Neurobiology Unit and

\section{Introduction}

TBC1D24 mutations are involved in a variety of neurological autosomal recessive disorders, including benign epilepsies, severe developmental encephalopathies with epilepsy and a complex neurological syndrome with Deafness, Onycodystrophy, Osteodystrophy, Mental Retardation and Seizure (DOORS syndrome) [1]. Additional TBC1D24 mutations have also been described in non-syndromic

Laboratories, Children's Hospital A. Meyer-University of Florence, Florence, Italy

4 INMED, Aix-Marseille University, INSERM U1249 Marseille, France

IRCCS Ospedale Policlinico San Martino, Genoa, Italy

6 Center of Synaptic Neuroscience and Technology, Istituto Italiano di Tecnologia, Genoa, Italy 
deafness with either dominant or recessive mode of inheritance [2-4].

$T B C 1 D 24$ encodes for a protein with two highly conserved domains namely a TBC domain, shared by several Rab GTPase Activating Proteins (GAP), and a TLD domain, implicated in oxidative stress resistance [5]. The Drosophila TBC1D24 homologue Skywalker acts as a GAP for Rab35 and binds phosphoinositides, thus playing a role in synaptic vesicle trafficking at larva neuromuscular junctions [6-8]. In the murine brain, TBC1D24 participates in cortical neuron maturation through the regulation of small GTPase ADP-ribosylation factor 6 (Arf6) [9-11]. Recently, a role in cell protection from oxidative stress, shared with the other TLDc containing proteins, has been also proposed [5, 12].

Pathogenic mutations span the entire TBC1D24 coding sequence, although no genotype/phenotype correlation has emerged so far from a systematic analysis of TBC1D24 patients [1]. Within the wide spectrum of neurological disorders associated with TBC1D24 mutations, developmental delay and early onset seizures are the most recurrent phenotypes. How the multiple functions of TBC1D24 impact on early neuronal development and excitability, and how they are related to the spectrum of pathologies associated with TBC1D24 dysfunction is a matter of investigation. Here we modelled TBC1D24 loss of function mutations by silencing TBC1D24 expression in developing rat cortical neurons in vitro and in vivo and identified a defect in axonal specification and projections. The axonal phenotype resulted in altered excitability and was accompanied by Arf6 dysregulation and defective membrane trafficking at the growth cone (GC). The same phenotype was reproduced in neurons differentiated from human induced pluripotent stem cells (hiPSCs) of a previously described patient with severe epileptic encephalopathy [1], but not in hiPSC-derived neurons from a patient with the milder phenotype of familial infantile myoclonic epilepsy (FIME) [9]. The results demonstrate axonal polarization and functional defects in neurons lacking TBC1D24 that are likely to contribute to the developmental phenotype in patients with severe epileptic encephalopathy.

\section{Results}

\section{TBC1D24 is highly expressed during the early phases of neuronal differentiation}

To evaluate the expression profile of TBC1D24 during the early phases of neuronal differentiation, we performed western blot analysis from rat primary cortical neurons $(\mathrm{PCN})$ at various developmental stages (0-10 days in vitro,
DIV). We detected an increase in TBC1D24 expression during the first stages of differentiation, in agreement with the previously reported expression profile in the rat cerebral cortex which progressively increases from embryonic to early postnatal stages [10]. Interestingly, TBC1D24 expression was virtually absent in twin astrocyte cultures, suggesting that, in the central nervous system, TBC1D24 is mainly neuron-specific (Fig. 1a).

\section{TBC1D24 loss impairs axonal specification and action potential firing}

To evaluate the effect of TBC1D24 loss of function at early stages of neuronal differentiation, we nucleofected PCN the day of preparation (0 DIV) with either the previously characterized sh-RNA targeting the $3^{\prime}$-untranslated region of rat TBC1D24 mRNA (Sh-TBC) [10] or its scrambled version (Scr-TBC), together with a GFP reporter to visualize transfected cells. Western blot analysis at 4-5 DIV revealed a net decrease in TBC1D24 expression in Sh-TBC transfected neurons with respect to either non-transfected or Scr-TBC transfected cells (Fig. 1b). Considering that $34.1 \pm$ $6.8 \%(\operatorname{Scr}-\mathrm{TBC} n=8)$ and $40.7 \pm 6.9 \%(\mathrm{Sh}-\mathrm{TBC} n=8)$ of neurons were nucleofected, the observed overall decrease of TBC1D24 immunoreactivity in total cell lysates likely represents a complete loss of expression in single transfected cells. Morphological analysis at the same developmental stage revealed a strong reduction of total neurite length, with a selective impairment of the putative axon evaluated by both Sholl analysis and lenght of the longest process (Fig. 1c, d). We also performed viability assays on Scr-TBC and Sh-TBC GFP-positive transfected cells, in view of the recently reported effect of TBC1D24 on the resistance to oxidative stress [12]. No differences were observed in the number of both pyknotic nuclei and caspase- 3 positive cells, suggesting that neurite elongation defect was a developmental effect, rather than a consequence of increased cell susceptibility to death (Suppl. Figure 1).

To directly address axonal specification in TBC1D24silenced cultures, we labelled 4-5 DIV transfected neurons with the axonal marker SMI-312, directed against highly phosphorylated axonal epitopes of neurofilaments, that represent one of the earliest recognizable markers of maturing axons. We found that only a small percent of GFP-positive neurons were expressing SMI-312 in TBC1D24-silenced neurons as compared to control cultures. Interestingly, the effect was significantly rescued by the concomitant expression of human TBC1D24, indicating that the observed phenotype was a consequence of TBC1D24 loss (Fig. 2).

To better characterize the axonal defect, we evaluated the generation of the axon initial segment (AIS), a structurally 
A
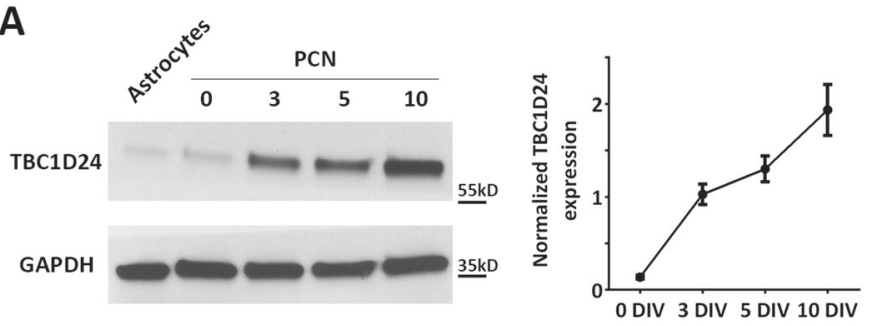
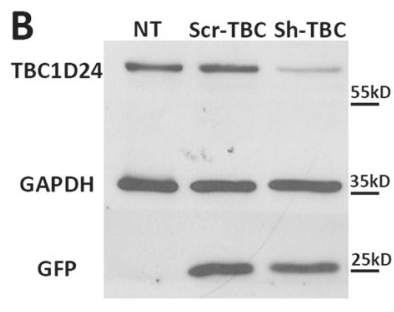

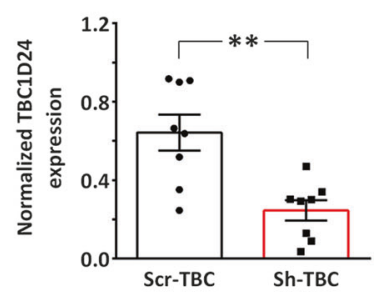

C
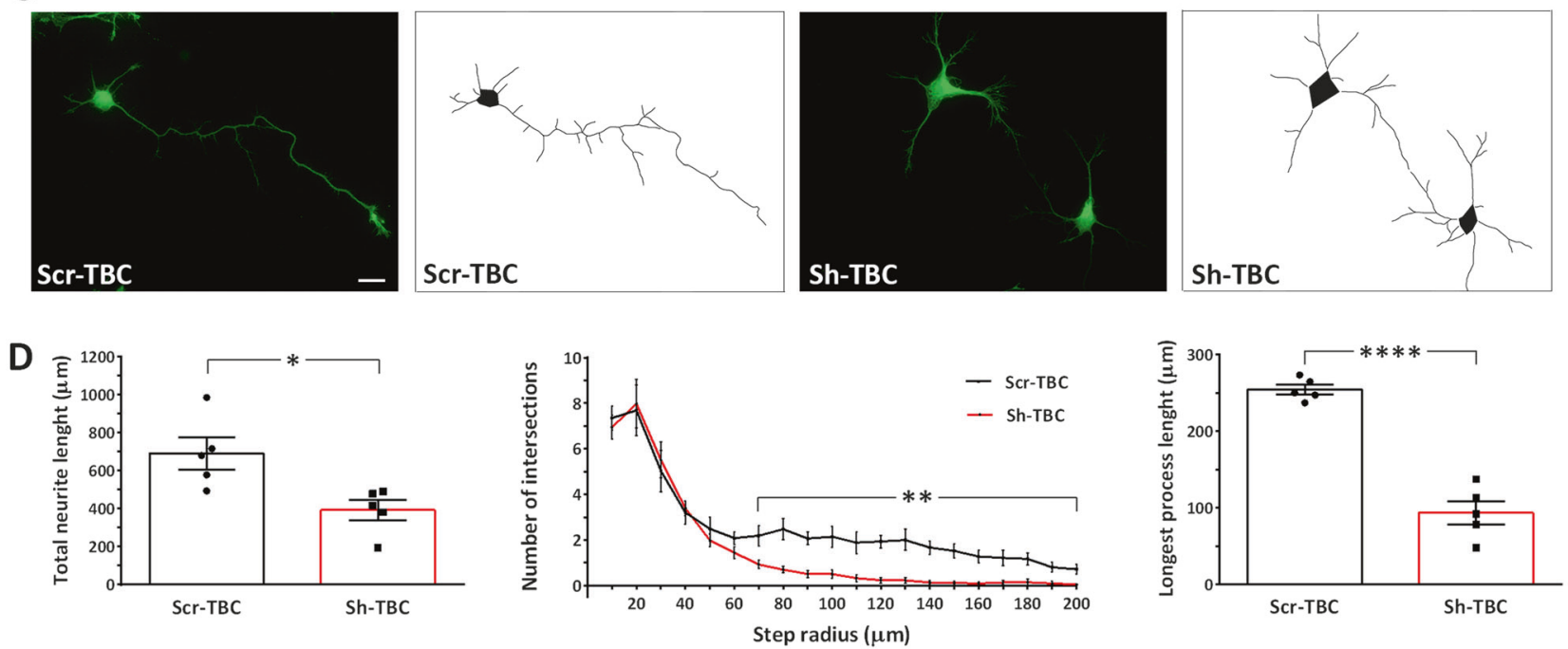

Fig. 1 TBC1D24 expression increases in PCN at early stages of development and its silencing leads to a reduced neurite arborisation. a Representative western blot analysis showing TBC1D24 expression in rat PCNs at various stages of differentiation and in twin astrocytic cultures. Densitometric quantification with respect to time 0 is shown in the line plot on the right. Data are means \pm SEM of 5 experiments from 3 independent preparations. b Representative western blot analysis showing the effective TBC1D24 silencing in rat PCNs after 5 days of transfection. Densitometric quantification is shown on the right. GAPDH is shown for equal loading and used for normalization.

specialized axonal domain crucial for polarization and action potential firing. AIS formation was investigated by labelling silenced and control neurons with antibodies to the specific markers Ankyrin-G (AnkG) and sodium channels (pan $\mathrm{Na}_{\mathrm{v}}$ ). As at 4-5 DIV the expression of AIS markers was almost undetectable, PCN were nucleofected at 0 DIV and analyzed for AnkG and $\mathrm{Na}_{\mathrm{v}}$ expression at later developmental stages (10 DIV). A slightly smaller percentage of TBC1D24-silenced neurons was positive for AnkG compared to scrambled-transfected controls (Fig. 3a, b). The mild defect in AnkG expression was accompanied by a dramatic reduction in the percentage of neurons expressing $\mathrm{Na}_{\mathrm{V}}$ (Fig. 3a, b), in line with previous works showing that the build-up of AIS sequentially starts from the AnkG-based scaffold and proceeds to recruit and cluster sodium channels [13]. Quantitative analysis of fluorescence intensity in neurons positive for both pan $\mathrm{Na}_{\mathrm{v}}$ and AnkG immunoreactivity did not reveal any significant differences (Fig. 3c, d). However, signal profiling revealed a longer AnkG-
GFP is shown as reporter of positive transfection. Data are means \pm SEM from 8 independent preparations. c Representative images of control and TBC1D24-silenced GFP-positive PCNs transfected at 0 DIV and analyzed at 5 DIV. The respective manual tracings are shown on the right of each image. Scale bar, $20 \mu \mathrm{m}$. d Total neurite length (left), Sholl analysis (center) and measurement of the longest neurite (right) of control and TBC1D24-silenced neurons treated as in C. Data are means \pm SEM from 5 independent preparations ( 88 and 93 neurons were analyzed for Scr-TBC and Sh-TBC respectively). Student' $t$ test: $* p<0.05 ; * * p<0.005 ; * * * * p<0.0001$

positive AIS and a distal shift of the maximum intensity for both AnkG and pan $\mathrm{Na}_{\mathrm{v}}$ immunoreactivities (Fig. 3e). Altogether, these data suggest that the AIS specification is impaired in TBC1D24 silenced neurons, with the majority of the neurons lacking the AIS, and the remaining neurons displaying an immature AIS characterized by distally misplaced markers.

To verify whether the observed defects in axonal specification resulted in a functional defect of action potential (AP) firing, we performed current-clamp recordings on 10 DIV PCN under the same experimental conditions used to evaluate the AIS development. We depolarized the membrane potential with successive current steps and observed a significant reduction of the instantaneous firing frequency and an increase of the current threshold to evoke AP firing (Fig. $4 \mathrm{a}-\mathrm{c}$ ). No other major changes in AP parameters were observed (Suppl. Table 1). To specifically gain information on AP firing at the AIS, we analysed the membrane potential change rate (i.e., dV/dt) versus voltage (phase- 
A

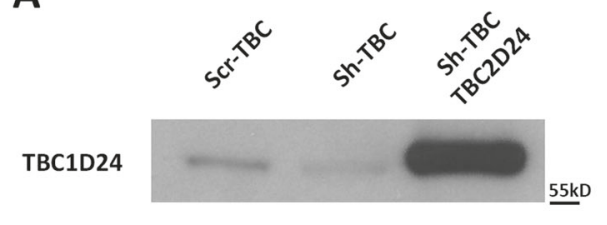

GAPDH
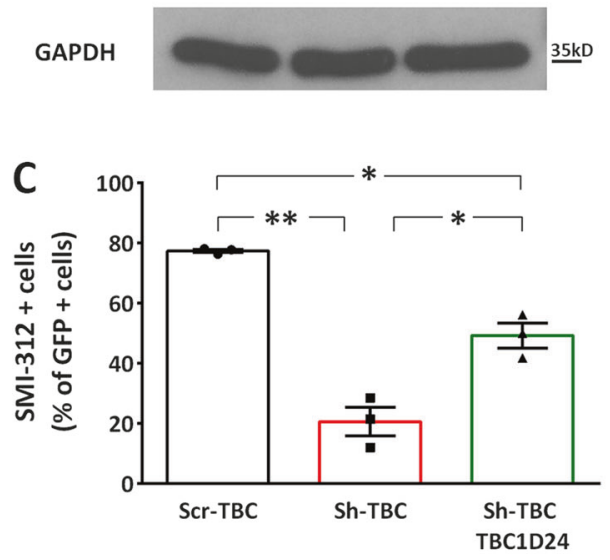

Fig. 2 Silencing of TBC1D24 impairs axon specification. a Representative western blot showing overexpression of TBC1D24 and its resistance to the Sh-TBC. GAPDH is shown for equal loading. b Representative images of transfected GFP-positive PCNs labelled for the axonal marker SMI-312 (red). Scale bar $20 \mu \mathrm{m}$ ). c The frequency

plane plot), where the first component (termed kink) is due to the spike initiation in the AIS and the second component represents the subsequent activation of sodium channels in the somato-dendritic compartment. This analysis showed significant differences in the kink phase between control and TBC1D24-silenced neurons. In particular, the 'slopekink', estimated by measuring the slope of the phase-plane plot using linear regression of the first 10 data points of the rising phase with a Y-axis value higher than $5 \mathrm{mV} / \mathrm{ms}[14$, 15], was reduced in the absence of TBC1D24 (Fig. 4d-f). These results suggest that TBC1D24 silencing, affects AP firing at both somatic and AIS sites.

\section{TBC1D24 loss impairs axonal projections in vivo}

To test whether TBC1D24 knockdown impairs axonogenesis in vivo we introduced Sh-TBC or Scr-TBC in combination with a GFP-encoding reporter construct into neuronal progenitor cells by in utero electroporation (IUE) at E15 and analyzed callosal projection in P7 brains. At this developmental stage, we previously reported no obvious alterations in cortical layering with impaired dendritic arborization [10]. We identified corpus callosum (cc) axon bundles in coronal cortical sections with SMI-312 labelling and quantified the ratio of GFP intensity in the cc versus the respective signal in the somatosensory cortex $(\mathrm{Cx})$. A significant reduction in cc/Cx ratio was observed in Sh-TBC electroporated brains with respect to Scr-TBC and rescued by concomitant expression of TBC1D24 (Fig. 5). The axonal projection phenotype was already evident at perinatal stage (P0, Suppl. Figure. 2), but at this stage many transfected neurons were still ectopic, as previously observed at embryonic stage [10]. Considering that cortical neurons start to project during migration $[16,17]$, these data also suggest a prominent role of TBC1D24 in axonal specification in vivo.

\section{The axonal phenotype is associated with Arf6 dysregulation and defective membrane trafficking at the growth cone}

The TBC1D24 molecular partner Arf6 plays important roles in neurite outgrowth and axonal specification [18-20]. We previously demonstrated that the dominant-negative locked form of Arf6 (Arf6-T27N) rescues the neuronal migration and dendritic outgrowth defects induced by in vivo TBC1D24 knockdown [10]. We therefore asked if the regulation of Arf6 was involved in the loss of axonal specification observed upon TBC1D24 silencing. We found that the level of GTP-bound Arf6 was significantly increased in 5 DIV neurons silenced for TBC1D24 as revealed by immunoprecipitation assays (Fig. 6a). Moreover, when Arf6-T27N was concomitantly expressed with Sh-TBC, the defect in axonal specification induced by TBC1D24 silencing was partially rescued (Fig. 6b-d), suggesting that the effects of TBC1D24 silencing on axon 

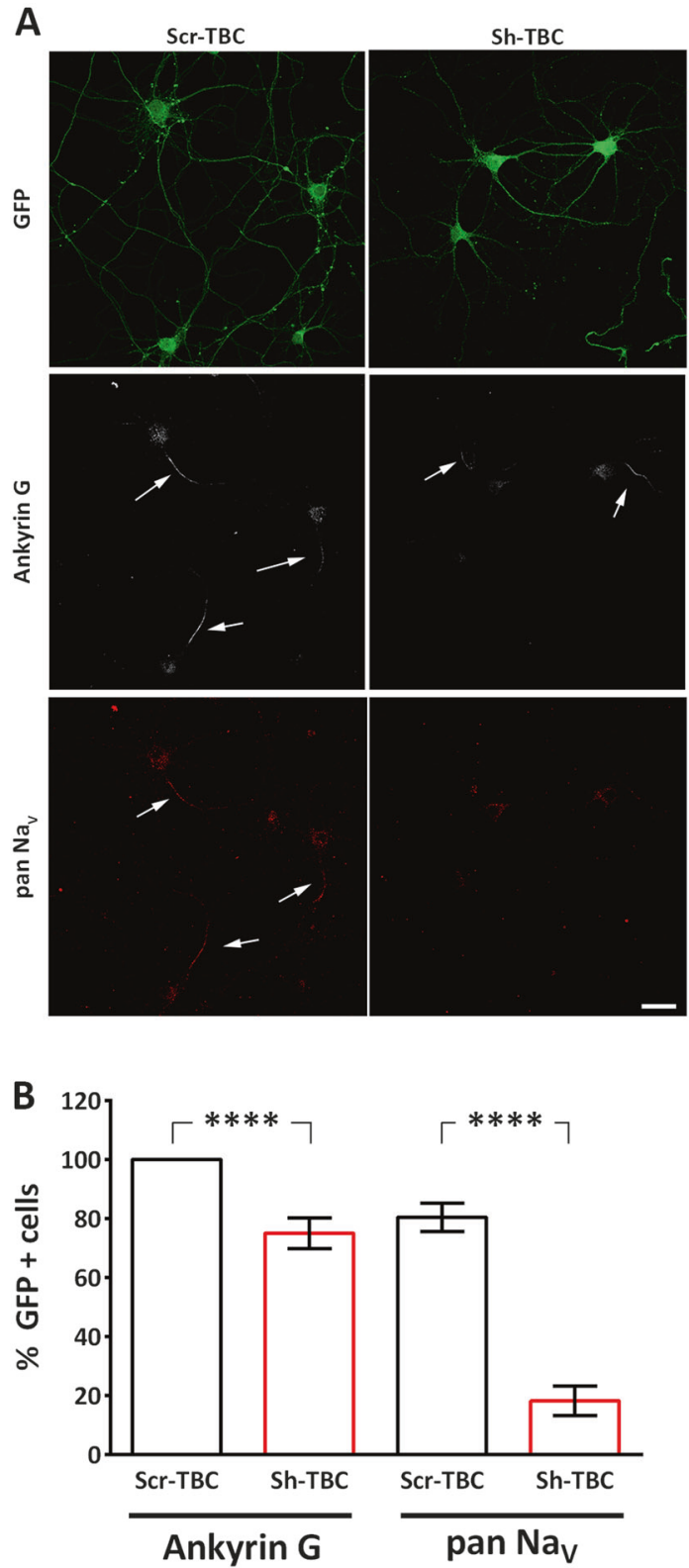

Fig. 3 Silencing of TBC1D24 impairs AIS formation. a Representative images of transfected GFP-positive PCNs labelled for the AIS markers Ankyrin $\mathrm{G}$ (grey) and pan $\mathrm{Na}_{\mathrm{V}}$ (red) at $10 \mathrm{DIV}$. White arrows indicate immuno-positive AIS tracts. Scale bar, $20 \mu \mathrm{m}$. b Quantification of the number of Ankyrin $\mathrm{G}$ and pan $\mathrm{Na}_{\mathrm{V}}$-positive cells expressed in percent on the total number of GFP-positive cells. Data are means \pm SEM from 46 and 54 fields for Scr-TBC and Sh-TBC respectively, from 3 independent preparations. $\mathbf{c}$ High magnification of cells treated as in A. Scale bar, $5 \mu \mathrm{m}$. d Quantification of the Ankyrin $\mathrm{G}$ and pan $\mathrm{Na}_{\mathrm{V}}$

specification are in part mediated by dysregulation of Arf6 activation.

Axon growth in the mammalian CNS is achieved by a complex and fine coordination of cytoskeletal rearrangement and vesicular trafficking processes [21] in which Arf6 has been shown to play a role $[19,22]$. We therefore tested whether TBC1D24 silencing had an influence on actin
C
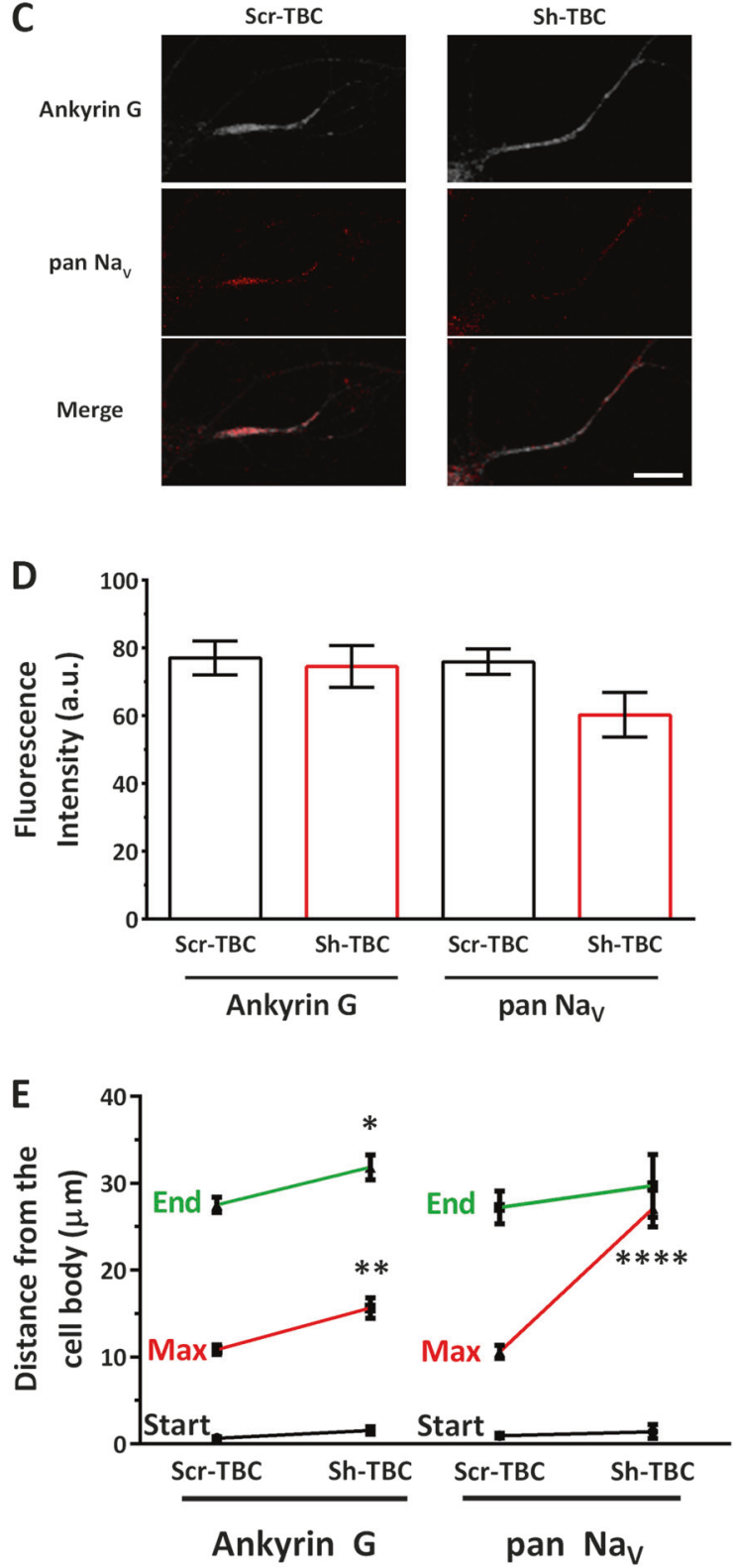

fluorescence intensity in immuno-positive control and TBC1D24silenced neurons. e Comparative measurements of the AIS start, maximum and end distances from the cell body performed for both Ankyrin $\mathrm{G}$ (left) and pan $\mathrm{Na}_{\mathrm{V}}$ (right) immunostainings in control and TBC1D24-silenced neurons. Data are expressed as means \pm SEM from 92-61 Ankyrin $\mathrm{G}$ and 71-13 pan $\mathrm{Na}_{\mathrm{V}}$ for Scr-TBC and Sh-TBC, respectively, in 3 independent preparations. Among GFP-positive neurons, Student's $t$ test: $* p<0.05 ; * * p<0.005 ; * * * p<0.0001$

dynamics by measuring the morphology of F-actin structures with phalloidin staining. Actin protrusions at distal neurite tips were measured by subtracting the $\beta$ III-tubulin immunofluorescent areas from total phalloidin-stained areas [23]. However, no differences were detected in actin protrusion areas between control and TBC1D24-silenced neurons (Suppl. Figure 3A,B). The lack of effect of 
A

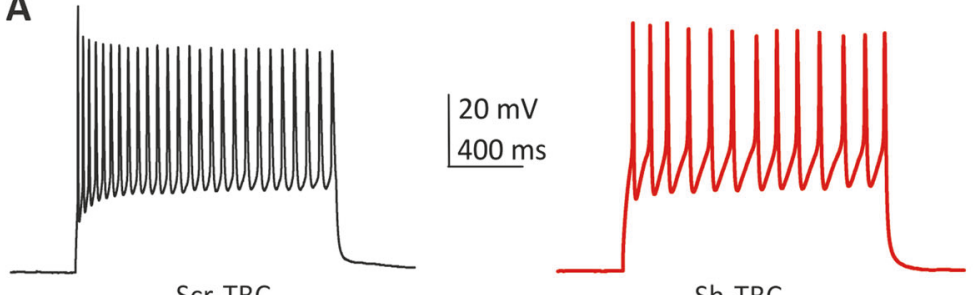

Scr-TBC
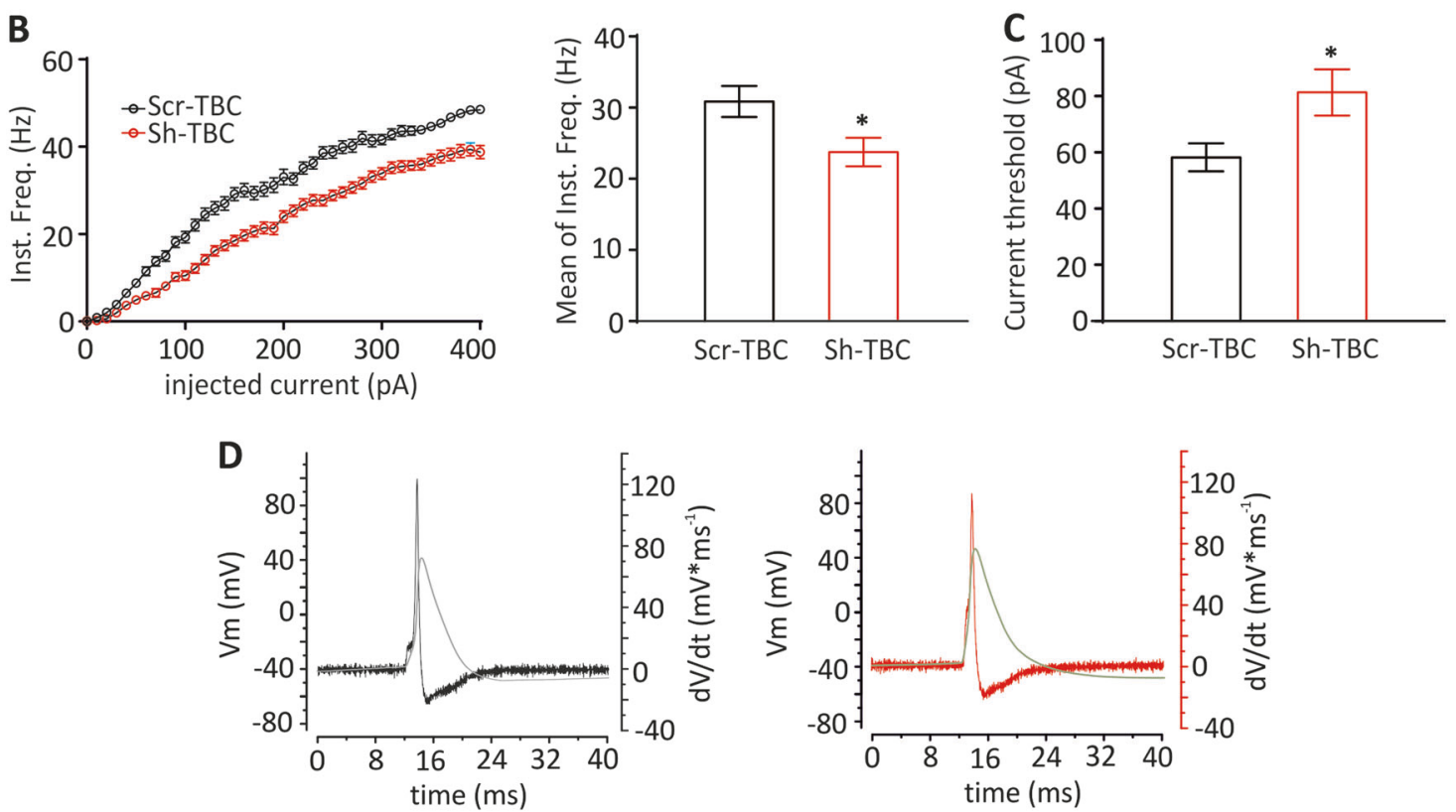

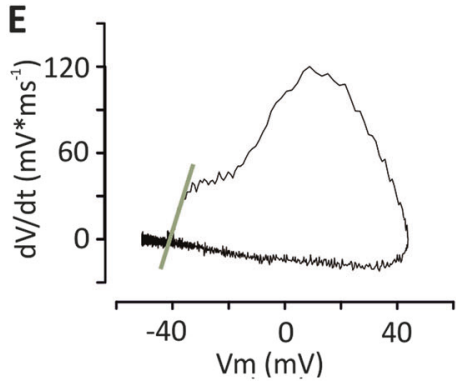

Fig. 4 Silencing of TBC1D24 alters neuronal excitability. a Representative current-clamp recordings of spike trains evoked by somatic current injection of $+180 \mathrm{pA}$ for $1 \mathrm{sec}$ in GFP-positive neurons transfected with a Scr-TBC (black) and Sh-TBC (red). b Graphs showing instantaneous firing frequency versus injected current (left) and their averaged values (right). c Graphs showing current threshold. Data are means \pm SEM of 29-32 cells from 2 independent preparations. d Representative action potentials (black and red traces for control and

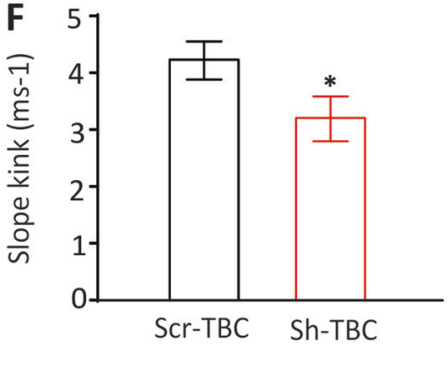

TBC1D24-silenced neurons, respectively) superimposed to the time course of the first derivative of the membrane voltage $(\mathrm{dV} / \mathrm{dt}$, grey traces). e Representative phase-plane plots of the first derivative of the membrane voltage $(\mathrm{dV} / \mathrm{dt})$ versus membrane voltage $\left(\mathrm{V}_{\mathrm{m}}\right)$. The gray line represents the linear regression of the first 10 data points of the rising phase with a Y-value $>5 \mathrm{mV} / \mathrm{ms}$, used to calculate the slope of the kink at the action potential threshold. f Slope of the phase-plane kink at the action potential threshold. Student's $t$ test: * $p<0.05$

TBC1D24 silencing on actin cytoskeleton was confirmed by parallel experiments of protein fractionation, in which no difference in $\mathrm{G} / \mathrm{F}$ actin ratio was found between control and TBC1D24-silenced neurons (Suppl. Figure 3C). Considering the additional role of microtubule dynamics in axonogenesis [24], we also investigated the accumulation of acetylated $\alpha$-tubulin in control and TBC1D24-silenced neurons, but again no differences were found at both cell body and axon levels (Suppl. Figure 4A-C). This experimental evidence prompted us to exclude a major involvement of cytoskeletal remodeling in the effects of TBC1D24 silencing on axonal specification and outgrowth.

Next, we investigated whether membrane trafficking defects were involved in the establishment of the axonal phenotype. We first asked whether TBC1D24 is present in the GC, the major site for membrane addition during axonal 
A

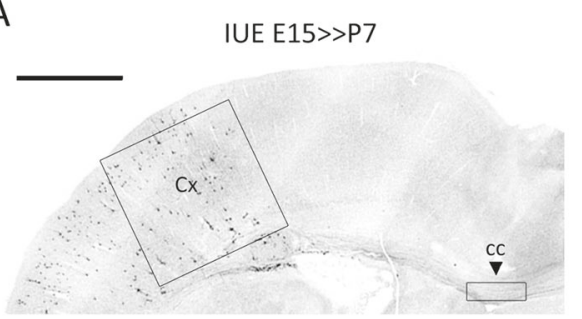

C

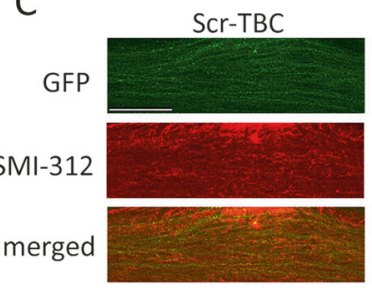

B
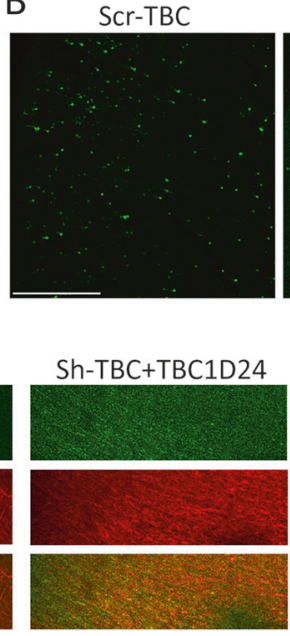

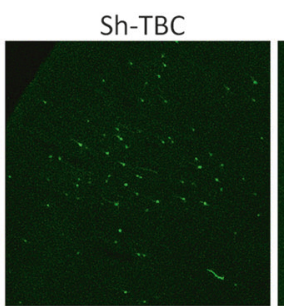

Sh-TBC+TBC1D24
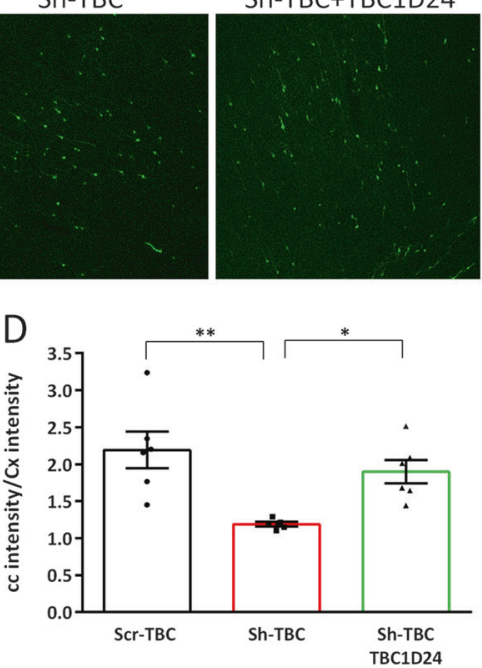

Fig. 5 Silencing of TBC1D24 leads to an axonal projection defect. a Representative coronal cortical section from P7 rat brain electroporated at E15 with GFP. Boxes show areas used to calculate fluorescence intensity of neurons in the somatosensory cortex $(\mathrm{Cx})$ and their projections extending toward the corpus callosum (cc). Scale bar, $1 \mathrm{~mm}$. b Representative P7 somatosensory cortical sections showing GFP-positive cells in brains electroporated at E15 with the indicated plasmids. Scale bar, $500 \mu \mathrm{m}$. c High magnification of cc bundle under

elongation. Because of the lack of reliable antibodies to detect endogenous TBC1D24, we overexpressed TBC1D24-GFP and analyzed its subcellular localization in PCN at 5 DIV, the developmental stage at which axonal elongation was impaired. TBC1D24-GFP was expressed both at the cell body and in phalloidin-positive GCs, with an enriched signal in the GC core (Fig. 7a) and co-localized with Arf6 at the GC when the two proteins were concomitantly overexpressed (Fig. 7b). To address whether TBC1D24 silencing altered endocytic membrane trafficking, we employed the styryl dye FM4-64, which has been reported to efficiently measure constitutive endocytosis in the GC at early stages of neuronal differentiation [25]. Nucleofected neurons were incubated for 1 min with FM464 , fixed and analyzed for the fluorescence intensity at the GC. While the FM4-64-positive GC area was not affected by TBC1D24 silencing, the mean FM4-64 fluorescence intensity at the GC was significantly lower, indicating a decreased FM4-64 uptake due to a defective endocytic membrane trafficking. The defective endocytic process observed at the GC was rescued by concomitant expression of TBC1D24 (Fig. 7c-e).

\section{hiPSC-derived neurons from TBC1D24-EOEE patient show axonal specification impairment}

We next asked whether the observed axonal phenotype could play a role in the establishment of the pathological the same experimental conditions as in B showing GFP signal, SMI312 labellling and their merge. Scale bar, $100 \mu \mathrm{m}$. d Quantification of the cc axon bundle GFP fluorescence intensity normalized to the respective GFP intensity in the ipsilateral $\mathrm{Cx}$. Data are means \pm SEM from 6 (Scr-TBC), 5 (Sh-TBC) and 6 (Sh-TBC + TBC1D24) brains obtained from two independent electroporation. $* p<0.05$, $* * p<$ 0.001; Kruskal-Wallis/Dunn's tests

phenotype by analyzing axonal development in patientderived neuronal cells. We obtained fibroblasts from skin biopsies of two previously described patients that are representative of mild and severe epileptic phenotypes (Suppl. Table 2). The first one (Patient 1, PAT 1) is affected by FIME and bear a heterozygous compound mutation (p. Asp147His/Asp509Val); [9] the second one (Patient 2, PAT 2) is affected by Early Onset Epileptic Encephalopathy (EOEE) associated with a homozygous missense mutation (p.Asp11Gly) [1]. Fibroblasts from two healthy sex- and age-matched individuals were used as controls (CTR1 and CTR2). We established multiple feeder-free lines of transgene-free hiPSCs from each individual, as previously described [26]. Sanger sequencing of the generated hiPSC lines confirmed the presence of the mutations in both patients (Suppl. Figure 5A); array-CGH assays showed the absence of genomic rearrangements in all hiPSC lines (Supplementary Figure 5B) and the stem cell pluripotency state of all hiPSC lines was also confirmed (Suppl. Figure 5C-D). We then differentiated controls' and patients' hiPSCs into a pure and stable population of self-renewable neural precursor cells (NPCs), as schematically reported (Suppl. Figure 6A). NPC cultures from control and TBC1D24-hIPSCs were characterized as previously described [26] (Suppl. Figure 6B,C). To support the differentiation, NPCs were cultured on a rat cortical astrocyte feeder and despite astrocytes were propagated for different passages in order to deplete them from neurons, NPCs were 

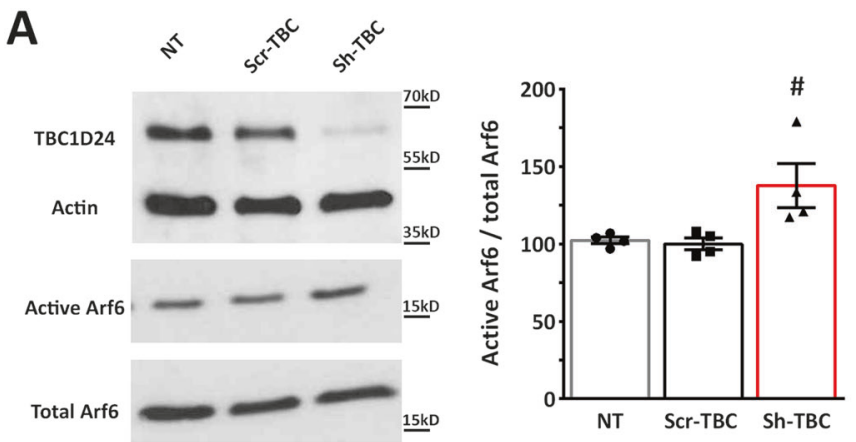

B
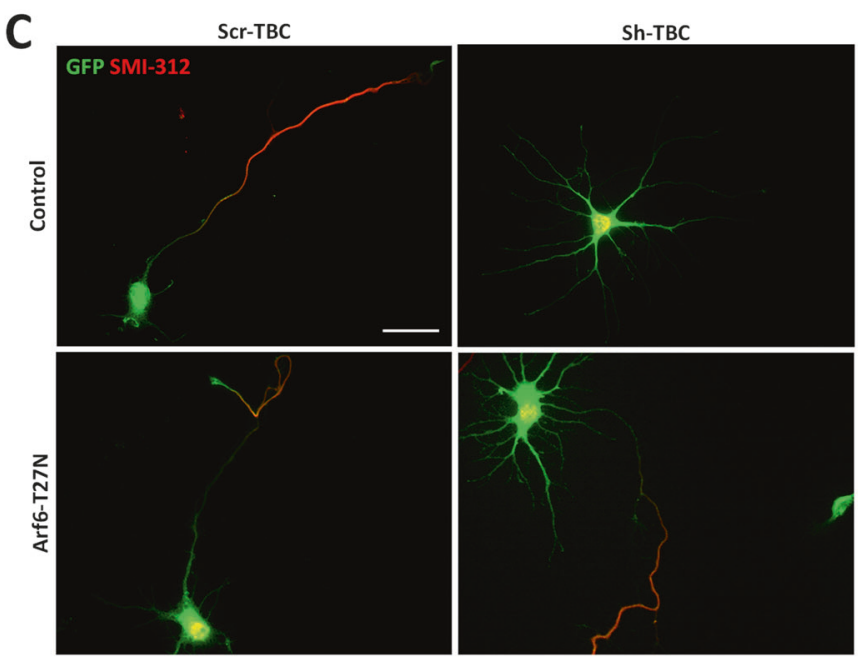

D
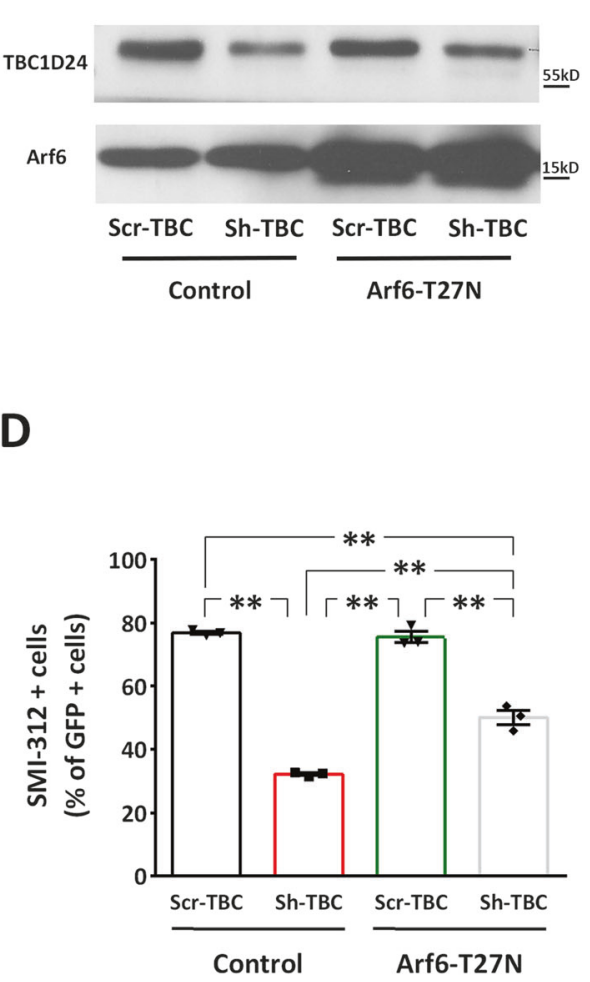

Fig. 6 An increased Arf6 activation is responsible for the defective axonal specification in TBC1D24-silenced neurons. a Representative western blot of active Arf6 immunoprecipitation from 5 DIV rat PCNs that were either untreated (NT) or transfected with Scr-TBC/Sh-TBC. Quantification of the Arf6-GTP normalized to total Arf6 is shown on the right. Data are means \pm SEM from 4 independent experiments. \# $p$ $<0.05$ with One-way ANOVA/Kruskal-Wallis' test b Representative immunoblot TBC1D24 and Arf6 expression in Scr-TBC or Sh-TBC

transfected neurons (5DIV) with or without concomitant Arf6T27N overexpression. c Representative images of GFP-positive neurons transfected as above and labelled for SMI-312 at 5 DIV. Scale bar, 20 $\mu \mathrm{m}$. d Quantification of SMI-312-positive cells with respect to the total number of GFP-positive cells from 3 independent preparations (90, 222, 202 and 224 neurons were analyzed for Scr-TBC, Sh-TBC, Scr$\mathrm{TBC}+$ Arf6-T27N and Sh-TBC + Arf6-T27N, respectively). Oneway ANOVA/Bonferroni's tests: ** $p<0.001$

infected with an EGFP-expressing lentivirus before plating to unambiguously discriminate hiPSC-derived neurons from the rat feeder (Suppl. Figure 6D). Already after 5 days of differentiation, hiPSC-derived neurons expressed neuronal markers such as $\beta$ III-tubulin and acquired a polarized neuron-like morphology (Suppl. Figure 6E). At the same time, hiPSCs-derived neurons started to exhibit a more complex subcellular compartmentalization for dendritic (MAP2) and axonal (SMI-312) markers. Virtually all MAP2-positive cells were also positive for the human nuclear marker, indicating that all the neuronal cells present in our cultures were of human origin (Suppl. Figure 6F,G).

To evaluate axonal specification in hiPSC-derived neurons, we measured the percent of SMI-312-positive axonal processes with respect to total MAP2-positive neurons at various days of differentiation. SMI-312-positive cells increased in control- and patient-derived neurons upon differentiation, demonstrating the developmental regulation of axon formation in human reprogrammed neurons
(Fig. 8). However, neurons derived from the severe EOEE patient bearing the homozygous p.Asp11Gly mutation (PAT 2) showed a decrease in the number of SMI-312positive cells at 10 days of differentiation, still present at 15 days of differentiation. On the contrary, neurons derived from the mild FIME patient bearing the p.Asp147His/ Asp509Val compound heterozygous mutation (PAT 1) were not significantly different from controls.

\section{Discussion}

Most of the pathogenic mutations identified in TBC1D24 are predicted to be loss-of-function [1] and in patients with DOORS or severe EOEE, a loss of expression was demonstrated in peripheral cells $[27,28]$. TBC1D24 is known to regulate dendritic development and synaptogenesis in vitro and in vivo models; [9, 10, 29] however, little is known on the effects of lack of TBC1D24 function in the 
A

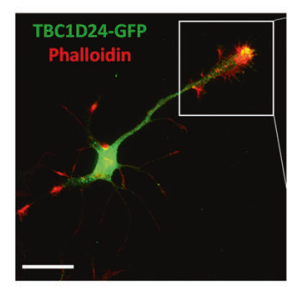

B

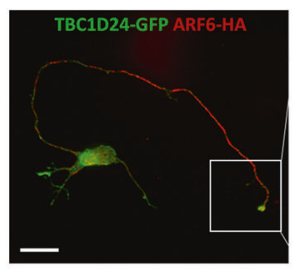

C
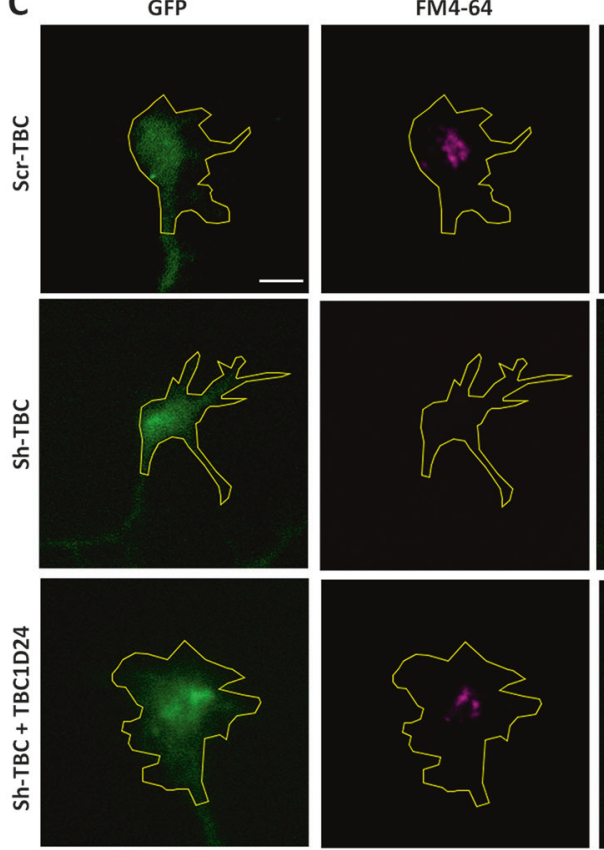

Fig. 7 Silencing of TBC1D24 results in membrane trafficking defect at the growth cone. a Representative images of 3 DIV rat PCNs transfected with TBC1D24-GFP (green) and labelled with Alexa Fluor 546 phalloidin (red) to visualize GCs. Scale bar, $20 \mu \mathrm{m}$. White square indicates the magnified images on the right show the enrichment of TBC1D24 at the GC central domain. b Representative images of 3 DIV rat PCNs co-transfected with Arf6-HA (red) and TBC1D24-GFP (green). Scale bar, $20 \mu \mathrm{m}$. White square indicates the magnified images

earlier stages of development, namely polarization and specification processes that are critical for the establishment of a functional axon. Here we demonstrate that silencing TBC1D24 in cortical neurons at early stages of differentiation impairs axon formation in vitro and callosal projections in vivo. The defective axon specification is associated with impaired maturation of the AIS and firing activity. The recapitulation of the defective axonal phenotype in the hiPSC-derived neurons prepared from severe EEOE patient, and not in neurons prepared from mild FIME patient, further suggest the relevance of TBC1D24 during
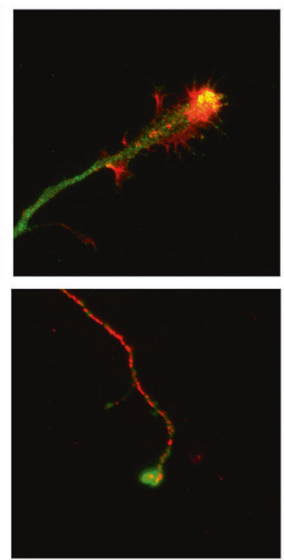

D
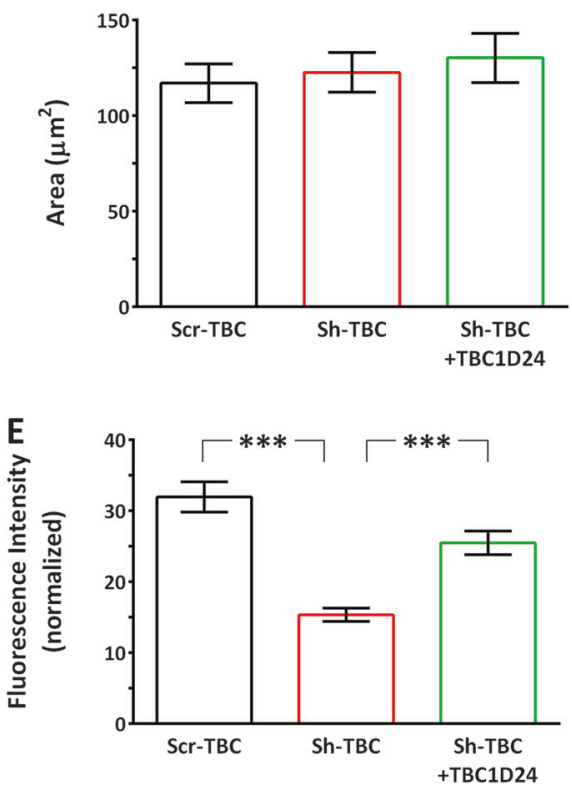

on the right showing colocalization of Arf6 and TBC1D24 at the GC. c Representative images of GFP-positive GCs from Scr-TBC, Sh-TBC and Sh-TBC/TBC1D24 transfected neurons after FM4-64 loading. Manual tracing of the GC area is shown. Scale bar, $5 \mu \mathrm{m}$. d Quantification of the GC area. e Quantification of FM4-64 fluorescence intensity normalized to the area analyzed. Data are means \pm SEM of 63-128 PCNs from 3 independent preparations. Kruskal-Wallis/ Dunn's tests: $* * * p<0.0001$

axon polarisation and its significant correlation with disease severity in humans. Notably, a thin corpus callosum has been reported in three TBC1D24 patients with severe epileptic phenotypes and additional axonal features, as myelination delay and white matter decrease, has been reported for other five patients with DOORS syndrome or severe myoclonic epilepsy [1]. The presence of immature neurons with altered firing properties in the neuronal network has been described for other genetic models associated with severe developmental encephalopathies with epilepsy [30-33]. 

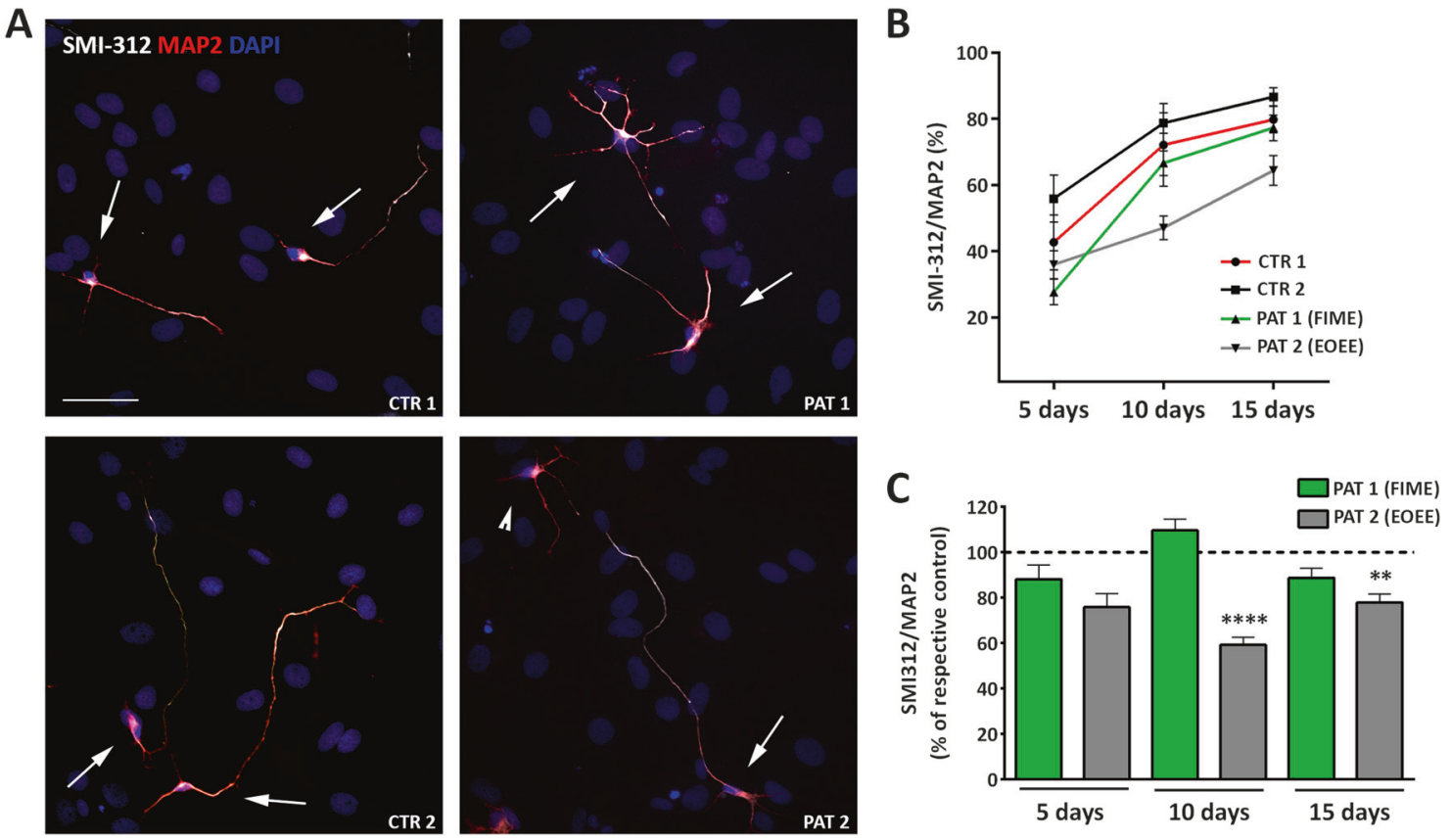

Fig. 8 hiPSC-derived neurons obtained from FIME and EOEE TBC1D24 patients show different axonal phenotypes. a Representative images of hiPSC-derived neurons at 10 days of differentiation from TBC1D24 patients (FIME, PAT 1 and EOEE, PAT 2) and controls (C1 and $\mathrm{C} 2$ ) were immunolabeled for SMI-312 and MAP2. Arrows indicate MAP2/SMI-312 double-positive neurons, while arrowheads indicate MAP2-positive/SMI312-negative neurons. Scale bar, $20 \mu \mathrm{m}$. b Representative experiments showing the developmental profile with percentages of SMI-312-positive cells in respect to the total MAP2-positive cells for hiPSC-derived neurons from TBC1D24

TBC1D24 plays diverse functions in neurons from the regulation of synaptic vesicle trafficking $[7,8]$ to the protection from oxidative stress [12] and interacts with several partners, such as small GTPases or phosphoinositides [7, 9, 11]. The axonal phenotype observed in TBC1D24-silenced neurons was not associated with increased cell mortality. Interestingly, it was accompanied by increased Arf6 activation (Arf6-GTP) and partially counteracted by expression of the dominant-negative Arf6 variant (Arf6-T27N), testifying an involvement of Arf6 dysregulation in the pathogenesis of the TBC1D24-related diseases. The activation state of Arf6 is reported to be highly regulated in developing neurons by multiple GTP Exchange Factors (GEFs) and GAPs, although the precise mechanisms of Arf6 cycling at the axonal GC are still debated [19, 20, 34]. We therefore propose that the TBC1D24 role in axon formation is, at least in part, attributable to its effect on the Arf6 activation state, as previously described for neurite elongation and arborization [10]. Several downstream effectors for active GTP-bound Arf6 exist that could play a role in axonogenesis [35], and among them the adaptor protein AP2 is of particular relevance [36]. Indeed, AP-2 deficiency causes impaired axonal extension, failure of sodium channel patients and relative controls. $\mathbf{c}$ Histograms showing the ratio of SMI312 on MAP2-positive hiPSCs-derived neurons with respect to respective control hiPSCs-derived neurons (dotted line) at various times of differentiation. Data are means \pm SEM of 4 experiments (two distinct clones from each experimental group). Fields analyzed for CTR 1, PAT 1, CTR 2, PAT 2, were respectively: 153, 211, 136, 212 (5 days of differentiation); 146, 209, 229, 199 (10 days of differentiation); 174, 92, 121, 120 (15 days of differentiation). KruskalWallis/Dunn's tests versus respective controls: $* * p<0.01$; **** $p<$ 0.0001

clustering at the AIS and defects in AP firing [37], resembling the TBC1D24 loss of expression phenotype described here.

Arf6 exerts multiple roles that are essential in early neuronal development, as it modulates axonal microtubule and actin dynamics [22] and protein trafficking [19]. Here, we demonstrate that the loss of TBC1D24 expression does not primarily affect cytoskeleton dynamics but results in membrane trafficking dysfunction. The axonal specification phenotype observed in TBC1D24 associates with a selective defect in membrane endocytic trafficking at the GC. The data suggest that, not only TBC1D24 governs SV trafficking at synaptic sites, but also regulates membrane trafficking in developing neurons. Interestingly, similar effects on membrane trafficking and increased Arf6 activation state were recently described in hair cells silenced for the Arf6 GAP ELMOD1 [38]. The involvement of TBC1D24 mutations in syndromic and non-syndromic deafness suggests an effect of TBC1D24 in endocytosis also within the cochlear system and opens new avenues for further research.

We recently described a mouse model of TBC1D24 haploinsufficiency and confirmed a role of mammalian 
TBC1D24 in neuronal development, protection from oxidative stress and SV trafficking [39, 40]. However, no significant effects were observed on callosal morphology, suggesting that, differently from acute knockdown, a partial and chronic loss of expression does not lead to a defect in axonal projections. The data are in agreement with the morpho-functional analysis on patients' hiPSC-derived neurons, that revealed an axonal defect only for the severe phenotype associated with a precocious TBC1D24 missense mutation, likely resulting in a complete loss of expression.

In conclusion, TBC1D24 is a pleiotropic gene with multiple functions in the brain. Its loss of function in developing neurons induces an axon specification defect associated with impaired outgrowth and maturation of the AIS region that alters neuronal excitability. This complex phenotype was observed both in TBC1D24-silenced rat neurons and hiPSC-derived neurons from patients bearing TBC1D24 mutations inducing a severe phenotype. The phenotype results from a global impairment of membrane trafficking due to Arf6 dysregulation that, at early stages of neuronal development, impairs the activity of the growth cone. Beyond the already described effects of TBC1D24 at synaptic level, these findings uncover a broader role of TBC1D24 in membrane dynamics and unveil a developmental pathomechanism for the severe encephalopathies associated with TBC1D24 loss of function mutations.

\section{Materials and methods}

\section{Antibodies and constructs}

The following primary antibodies were used at the indicated concentration: $\alpha$-tubulin (IC 1:1000, WB 1:10.000, T6074), acetylated $\alpha$-tubulin (IC 1:1000, WB 1:40.000, T7451), Actin (WB 1:5000, A4700), ßIII-tubulin (IC 1:2000, T2200), pan $\mathrm{Na}_{\mathrm{V}}$ (IC 1:100, S8809) and Arf6 (WB 1:1000, A5230) from Sigma-Aldrich; Ankyrin-G (IC 1:300, H-215) and GAPDH (WB 1:500, FL-335) from Santa Cruz; $\beta$-Actin (WB 1:10.000, 251 011), MAP2 (IC 1:500, 188 003) and GFP (IC 1:500, and IHC 1:250, 132 005) from Synaptic Systems; Caspase-3 (IC 1:100, AF835) from R\&D Systems; GFP (WB 1:1000, A11122) from Thermo Fisher Scientific; Human Nuclei (IC1:500, MAB 1281) from Millipore; SMI312 (IC 1:500, and IHC 1:250, 837904) from BioLegend; TBC1D24 (WB 1:1000, ARP57438_P050) from AVIVA; GFP (IC 1:500, MAB3580) from Millipore. Horseradish peroxidase (HRP)-conjugated secondary antibodies are from Bio-Rad. Fluorescent secondary antibodies (Alexa Fluor) are from Thermo Fisher Scientific. ShRNA targeting the 3'UTR coding sequence of Rattus norvegicus Tbc1d24 (Sh-TBC) and its negative control (Scr-TBC), pCAGIGIRES-EGFPpCAGIG-hTBC1D24-EGFP and pCAGIG-
ARF6 $^{\text {T27N }}$-EGFP vectors used for nucleofection were all previously described and validated in [10]. hiPSCs, cells from germ layers and NPCs were stained with anti-SSEA4, OCT4, SOX2, TRA-1-60, AFP, SMA, $\beta$ III-tubulin, Nestin, SOX1, Pax6 (all diluted 1:100) from Thermo Fisher.

\section{Cell culture and transfection}

Cultures of dissociated cortical primary neurons were prepared from embryonic E18 rats as previously described [41]. The embryonic cortices were dissected and incubated in $0,125 \%$ trypsin (Gibco) for $25-30 \mathrm{~min}$ at $37^{\circ} \mathrm{C}$. Dissociated neurons were plated onto poly-L-lysine-coated coverslips in a $25 \mathrm{~mm}$ glass coverslips or tissue culture dish for immunostaining and western blot, respectively. Neurons were transfected before plating by using the 4D Nucleofector System (LONZA) with the high viability protocol for primary rat neurons. For astrocyte preparation, cells obtained during primary cortical neurons preparation were plated on T75 flasks in the presence of Dulbecco's Modified Eagle Medium (Advanced DMEM) supplemented with $20 \%$ FBS, $1 \%$ Glutamine, $1 \%$ penicillin-streptomycin and maintained at $37{ }^{\circ} \mathrm{C}$ in a $5 \% \mathrm{CO}_{2}$ humidified atmosphere. Cells were propagated once $80 \%$ of confluence was reached and amplified until at least the third passage before use.

\section{In Utero Electroporation}

Wistar rats (Janvier Labs, France) were mated, cared for and used in our animal facilities in agreement with the European Union and French legislations. Timed pregnant rats (E15; E0 was defined as the day of confirmation of sperm-positive vaginal plug),received buprenorphine (Buprecare, $0.03 \mathrm{mg} /$ $\mathrm{kg}$ ) and were anesthetized with sevoflurane (4.5\%) $30 \mathrm{~min}$ later. The uterine horns were exposed, and a lateral ventricle of each embryo was injected using a picopump (PV 820 Pneumatic PicoPump; World Precision Instruments, Sarasota, FL) with Fast Green ( $2 \mathrm{mg} / \mathrm{ml}$; Sigma, St Louis, MO, USA) combined with the following DNA constructs: 0.5 $\mathrm{mg} / \mathrm{ml}$ pCAGGS-EGFP either alone or with $1.5 \mathrm{mg} / \mathrm{ml}$ of shRNA construct targeting the TBC1D24 mRNA. Plasmids were further electroporated by discharging a $4000 \mathrm{mF}$ capacitor charged to $40 \mathrm{~V}$ with a BTX ECM 830 electroporator (BTX Harvard Apparatus, Holliston, MA, USA). The voltage was discharged in five electrical pulses at 950 $\mathrm{ms}$ intervals via $5 \mathrm{~mm}$ electrodes placed on the head of the embryo across the uterine wall. Control and experimental embryos were obtained from the same litter and the injections were made on the right ventricles, for later identification. We performed in utero electroporation in embryonic rats at E15 corresponding to an active period of both radial and tangential migration of newborn neurons in the cortex. 


\section{Generation and maintenance of hiPSC lines}

hiPSCs were generated from dermal fibroblasts. Skin biopsies were performed upon informed consent using the punch biopsy procedure and fibroblasts were cultured in RPMI (Gibco) supplemented with $20 \%$ FBS, 2mM LGlutamine and $1 \%$ Penicillin/Streptomycin. TBC1D24 fibroblasts derive from two patients affected from FIME (PAT 1) and EOEE16 (PAT 2) respectively, while control fibroblasts -aged and sex matched (FF054/86 - C1 and FF085/91 - C2)- were obtained from the "Cell Line and DNA Biobank from Patients affected by Genetic Diseases" (Istituto G. Gaslini, Genova, Italy), a member of the Telethon Network of Genetic Biobanks (project no. GTB12101). The study was approved by the local Ethical Committee of the G. Gaslini Institute. Cells at low passages were reprogrammed by using the non-integrating Sendai virus (SeV) CytoTune ${ }^{\mathrm{TM}_{-} \text {iPS }} 2.0$ reprogramming Kit (Thermo Fisher Scientific) under feeder-free conditions according to the manufacturer's instructions. Colonies appeared after 25 days. At least 20 single colonies for each genotype were isolated by manual picking, maintained on recombinant human truncated Vitronectin (VTN-N)-coated plates in ESSENTIAL-8 flex culture medium (all from Thermo Fisher Scientific). Culture medium was refreshed every other day and cells were passaged using Versene solution every 3-5 days. Each clone was expanded separately as cell lines. At the 15 th passage, clones were tested for the expression of pluripotency markers by qRT-PCR and IF. For in vitro differentiation into cells of all three germ layers, confluent undifferentiated hiPSC were incubated in $1 \mathrm{mg} / \mathrm{ml}$ collagenase IV (Thermo Fisher) for $20 \mathrm{~min}$ at $37^{\circ} \mathrm{C}$ and transferred to $100 \mathrm{~mm}$ low attachment plates in Embryoid Bodies (EBs) medium (DMEM/ F12 supplemented with $20 \%$ knockout serum replacement, $1 \mathrm{mM}$ glutamine, $1 \%$ non-essential amino acids, $1 \%$ penicillin/streptomycin, $0.55 \mathrm{mM} \quad \beta$-mercaptoethanol, from Thermo Fisher Scientific). Cells, aggregated in free-floating EBs, were grown in suspension for 5-6 days in EB medium and then plated on Geltrex-coated dishes (Thermo Fisher) for further 5 days. The medium was changed every other day. To control for virus loss, standard PCR was performed using the primers recommended by the manufacturer. All hiPSC lines were mycoplasma-free, based on tests by the Mycoalert Mycoplasma Detection Kit (Lonza). At least two clones from each genotype were kept in culture for further analysis.

\section{Differentiation of hiPSC clones into neurons}

For neuronal differentiation, EBs were generated as described above and cultured for 5 days in suspension in EB medium. EBs were then collected and plated into a Geltrex- coated plates for additional 3-5 days to form neural tubelike rosettes in presence of neural induction medium consisting of DMEM/F12, N2 (1:100), supplemented with fibroblast growth factor 2 (FGF-2) $(20 \mathrm{ng} / \mathrm{ml}$; Thermo Fisher), SB431542 (10 $\mu$ M; Tocris) and LDN-193189 (0.1 $\mu \mathrm{M}$; Sigma Aldrich). The emerging neural rosettes were picked manually, dissociated completely using Accutase at $37{ }^{\circ} \mathrm{C}$ for $10 \mathrm{~min}$ and transferred to Geltrex-coated plates in NPC medium consisting of DMEM/F12 supplemented with N2 (1:100), B27 (1:500; GiBCO), epidermal growth factor (EGF; $20 \mathrm{ng} / \mathrm{ml})$ and FGF-2 $(20 \mathrm{ng} / \mathrm{ml})$. NPCs were expanded and fed every other day. To obtain terminally differentiated neurons, NPCs infected with Lentiviral vector 743.pCCLsin.PPT.hPGK.GFP.Wpre (a gift from Dr. L. Naldini) were plated at the concentration of $1.5 \times 10^{4}$ cells/ $\mathrm{cm}^{2}$ onto Geltrex-coated round glass coverslips in coculture with $0.8 \cdot 10^{4} / \mathrm{cm}^{2}$ rat astrocytes. Neuronal differentiation lasted 15-20 days in Neurobasal-A medium supplemented with L-glutamine $(2 \mathrm{mM}), \mathrm{N} 2$ (1:100), B27 (1:50), BDNF $(10 \mathrm{ng} / \mathrm{ml})$, GDNF $(10 \mathrm{ng} / \mathrm{ml})$ (all from Thermo Fisher) and retinoic acid ( $1 \mu \mathrm{M}$; Sigma-Aldrich). Half of the medium was replaced every other day during continuous culturing.

\section{DNA genotyping and copy number variations (CNVs) analysis}

The presence of TBC1D24 mutations was assessed by the standard Sanger method. Genomic DNA was extracted from cultured fibroblast and hiPSC lines using the QIAmp DNA mini kit (Qiagen) and sequence analysis was performed using a $3130 \mathrm{xl}$ Genetic Analyzer (AB Applied BiosystemsHitachi). Analysis of CNVs was performed by Array-CGH using Human Genome CGH Microarray kit $8 \times 60 \mathrm{~K}$ (Agilent $^{\mathrm{TM}}$, Agilent Technologies, Santa Clara, CA) following to manufacturer's protocol. Genomic DNA from each hiPSC line and his parental fibroblast were labelled with Cyanine3/Cyanine 5 and competitively hybridized on a CGH-array. Data were analysed using the Cytogenomics software with the following settings: aberration algorithm ADM-2; threshold 6.0; window size $0.2 \mathrm{Mb}$; filter 4 probes, DLRS $<0.25$.

\section{qRT-PCR}

qRT-PCR was performed in a real-time thermal cycler (CFX-96, Bio-Rad) using SsoFastEvaGreen Supermix (BioRad). Total mRNA was extracted with the RNeasy mini kit (Qiagen) according to the manufacturer's instructions, and reverse transcribed using the iScriptcDNA Synthesis Kit (Bio-Rad). All primers are listed in Suppl. Table 3. Relative expression was calculated based on the $2^{-\Delta \Delta \mathrm{Ct}}$ method [42] by normalizing data to the geometric mean of three 
housekeeping transcripts (GAPDH, PPIA, RPL13A) using the CFX Manager 3.0 software (Bio-Rad).

\section{Western blot analysis and pull-down experiments}

Cells were lysed in lysis buffer $(\mathrm{NaCl} 150 \mathrm{mM}$, EDTA 1 $\mathrm{mM}$, TrisNaCl $50 \mathrm{mM} \mathrm{pH} \mathrm{7.4,1 \%} \mathrm{Triton} \mathrm{X-100,} \mathrm{PMSF} 2$ $\mathrm{mM}$ and Pepstatin $1 \mu \mathrm{g} / \mathrm{ml}$ ). Proteins were quantified by Bradford protein assay (BioRad), loaded and resolved by SDS/PAGE and transferred onto nitrocellulose membranes (Whatman). After block with 5\% milk, membranes were incubated with primary antibodies, conjugated with HRPconjugated secondary antibodies and revealed with the ECL chemo-luminescence detection system (Thermo Fisher).

Experiments to evaluate the Arf6 activation state were performed using an Arf6-GTP-specific pull-down assay (Cytoskeleton, Denver, CO), as previously described [10]. For the G/F actin separation protocol, we adapted the protocol described in [22]. Neurons, collected after nucleofection, were washed three times in PBS at $37{ }^{\circ} \mathrm{C}$ and lysed for $10 \mathrm{~min}$ in Actin Stabilization buffer (0.1 M PIPES pH 6.9, 30\% Glycerol, 5\% DMSO, $1 \mathrm{mM} \mathrm{MgSO}_{4}, 1 \mathrm{mM}$ EGTA, $1 \%$ Triton X-100 supplemented with $1 \mathrm{mM}$ ATP, 2 $\mathrm{mM}$ PMSF and $1 \mu \mathrm{g} / \mathrm{ml}$ Pepstatin). Cells were dislodged by scraping and lysates centrifuged in Beckmann TL-100 centrifuge at $100,000 \times \mathrm{g}$. The supernatant containing Gactin was separated from the pellet containing F-actin and both suspended in Laemmli buffer for SDS-PAGE separation.

\section{Immunocytochemistry}

Neurons plated on glass coverslips were washed three times with $1 \mathrm{X}$ phosphate buffered saline (PBS, Sigma-Aldrich) at $37^{\circ} \mathrm{C}$ to remove culture medium and fixed by using $4 \%$ Paraformaldehyde (PFA, Sigma-Aldrich) and 4\% Sucrose (Applichem) in PBS at $37{ }^{\circ} \mathrm{C}$ for $15 \mathrm{~min}$. Excess of PFA was removed by washing with PBS and cells were permeabilized using $0.1 \%$ Triton X-100 in PBS for $5 \mathrm{~min}$. Non-specific epitopes were saturated with $2.5 \%$ fetal bovine serum (FBS, Gibco) in PBS. Primary antibodies diluted in PBS/2.5\% FBS were applied for $2 \mathrm{~h}$ at room temperature (RT) or overnight $(\mathrm{o} / \mathrm{n})$ at $4{ }^{\circ} \mathrm{C}$. The excess of antibody was removed by washing the coverslip three times with PBS and the secondary antibodies added for $45 \mathrm{~min}$ at RT. Alexa-Phluor ${ }^{\circledR} 568$ Phalloidin (diluted 1:35 in PBS; Thermo Fisher) was incubated for $45 \mathrm{~min}$ at RT and the excess removed with PBS. Coverslips were mounted by using Prolong Diamond antifade reagent (Thermo Fisher), containing 4', 6'-diamidino-2phenylindole (DAPI) to visualize nuclei.

For hiPSCs, three germ layer cells and neuronal precursor cells staining was performed with PSC 4-MARKER ICC KIT, 3-GERM LAYER ICC KIT and NSC ICC KIT respectively
(Thermo Fisher) according to the manufacturer's instructions. For the characterization of hiPSC-derived neurons, cells were fixed with $4 \%$ PFA for 15 min at RT, permeabilized with $0.1 \%$ Triton X-100 (Sigma) and blocked with $0.1 \%$ Triton X-100 with $10 \%$ FBS in PBS for 20 min. Samples were then incubated with primary antibodies at $4{ }^{\circ} \mathrm{C} \mathrm{o} / \mathrm{n}$, followed by incubation with secondary antibodies for $1 \mathrm{~h}$ at RT. Antibodies were prepared in PBS containing 0.1\% Triton X-100 and 5\% FBS serum. Images in epifluorescence were collected with an Olympus IX-81 microscope equipped with an MT20 Arc/Xe lamp, $\times 20, \times 40, \times 60$ objectives (Olympus) using the Excellence'RT software (Olympus). Confocal images were acquired by using a 40X objective in a Leica TCS SP8 Confocal Laser Scanning Microscope (Leica Microsystem). Images were processed and analyzed using the free software ImageJ (https://ima gej.nih.gov/ij/). Neurite length and Sholl analyses were performed using the ImageJ plugins NeuronJ and Sholl Analysis, respectively. The axon in developing neurons was defined by specific immunostaining of SMI-312. Actin protrusions were evaluated as described in [23]. Analysis on the AIS was performed as previously described [43].

\section{Electrophysiology}

Neuronal firing was studied by whole-cell current-clamp recordings. Recordings were performed using a Multiclamp 700B amplifier (Axon Instruments, Molecular Devices, Sunnyvale, CA) using an IX71 inverted phase/fluorescence (Olympus, Japan). The age of the patched neurons ranged between 10 and 12 DIV. Patch electrodes, fabricated from thick borosilicate glasses were pulled and fire-polished to a final resistance of 3-5 M $\Omega$. Current-clamp recordings of firing activity were performed in a standard external solution containing (in $\mathrm{mM}$ ): $140 \mathrm{NaCl}, 2 \mathrm{CaCl}_{2}, 1 \mathrm{MgCl}_{2}, 4$ $\mathrm{KCl}, 10$ glucose, 10 HEPES (pH 7.3 with $\mathrm{NaOH}$ ). The internal solution contained (in $\mathrm{mM}$ ): $126 \mathrm{~K}$-gluconate, 4 $\mathrm{NaCl}, 1 \mathrm{MgSO}_{4}, 0.02 \mathrm{CaCl}_{2}, 0.1$ BAPTA, 15 Glucose, 5 Hepes, 3 ATP, 0.1 GTP, $\mathrm{pH}$ 7.3. Current-clamp recordings were performed at a holding potential of $-70 \mathrm{mV}$, and action potential firing was induced by injecting current steps of $10 \mathrm{pA}$ lasting $1 \mathrm{sec}$. The parameters describing the action potential shape were analyzed using the pClamp software (Molecular Devices) and the Prism software (GraphPad Software, Inc.). For each recorded cell, the plot of the time derivative of voltage $(\mathrm{dV} / \mathrm{dt}$ ) versus voltage, called phaseplane plot, was obtained starting from the first action potential elicited by the minimal current injection $(10 \mathrm{pA}$ current steps). This parameter was used to better identify the voltage threshold and the maximum rising and repolarizing slopes [44]. The voltage threshold was defined as the first voltage value at which $\mathrm{dV} / \mathrm{dt}$ exceeded $10 \mathrm{mV} / \mathrm{ms}$. The slopes of phase-plots at the action potential threshold [15, 45], were calculated using linear regression of the first 10 
data points of the rising phase with a Y-value $>5 \mathrm{mV} / \mathrm{ms}$. The current threshold was calculated as the minimum depolarizing current needed to elicit at least one action potential. Instantaneous frequency (I-Freq) was calculated between the first two evoked action potentials for each step.

\section{Immunohistochemistry and axonal projections quantification}

For immunostaining rat brains were fixed (P0 or $\mathrm{P} 7)$ or perfused transcardially ( $\mathrm{P} 15)$ with $4 \%$ paraformaldeyde (PFA) in PBS. To make coronal sections, brains were cryoprotected by overnight immersion in $30 \%$ sucrose in PBS and embedded in OCT. Floating cryosections of 40$60 \mu \mathrm{m}$ were permeabilized with PBS containing $0.1 \%$ Triton X-100 and blocked with PBS containing $0.1 \%$ Triton X100 and 5\% FBS. The sections were incubated with primary antibody followed by Biotin-xx Goat anti-mouse IgGAb (B2763, Thermo), Streptavidin Alexa Fluor 647 and Hoechst33342 (Thermo) following the manufacturer instructions. Sections were imaged on a laser-scanning confocal microscope (SP4 Leica). Images were processed using ImageJ software. To evaluate axonal projections, we calculated the ratio between GFP fluorescence intensity in a $150 \times 600$ pixel rectangle at the cc axon bundles and the GFP fluorescence intensity in a $1500 \times 1500$ pixel in the corresponding ipsilateral cortex. Settings were kept the same for all acquisitions within each experiment.

\section{FM4-64 uptake}

3-5 DIV PCN cultures were washed two times in preheated Tyrode buffer $\left(\mathrm{NaCl} 140 \mathrm{mM}, \mathrm{KCl} 4 \mathrm{mM}, \mathrm{CaCl}_{2} 2\right.$ $\mathrm{mM}, \mathrm{MgCl}_{2} 1 \mathrm{mM}$, Hepes $10 \mathrm{mM}$, Glucose $10 \mathrm{mM}$ ). Coverslips were covered with $100 \mu \mathrm{l}$ of $10 \mu \mathrm{M}$ Fixable FM4-64 (Thermo Fisher) in Tyrode and incubated at $37^{\circ} \mathrm{C}$ for $1 \mathrm{~min}$. Cells were washed twice in Tyrode, immediately fixed with $4 \%$ PFA $/ 4 \%$ Sucrose in $1 \times$ PBS and mounted on glass support with Prolong Diamond anti-fade reagent. Analysis of the FM4-64-positive GCs was performed acquiring images at $\times 60$ of magnification. GC perimeters were manually traced on the image acquired in the GFP channel starting $2 \mu \mathrm{m}$ before the doubling of the axonal diameter at the beginning of each GC. Fluorescence intensity was calculated inside the perimeter after background subtraction by using a standard ROI placed nearby.

\section{Statistical analysis}

The normal distribution of experimental data was assessed using the D'Agostino-Pearson normality test. Data with normal distribution were analyzed by the unpaired Student's $t$ test or one-way analysis of variance (ANOVA) followed by the Bonferroni's multiple comparison tests. Nonnormally distributed data were analyzed by the KruskalWallis one-way analysis of variance on Ranks test followed by the Dunn's multiple comparison tests using Graphpad 7.0 (Graphpad Software Inc., La Jolla, CA). Significance level was preset to $p<0.05$. Data are expressed as means \pm SEM throughout for number of experimental sessions $(n)$.

Acknowledgements We would like to thank Dr. Anna Corradi for valuable discussion, Dr. Margherita Mancardi for clinical description of the patients, Sara Pepe for help with imaging analysis, Monica Traverso for genotyping and Silvia Casagrande for assistance in primary cell and plasmid preparation. This work is supported by the University of Genoa (FRA grant to A.F.), Compagnia di San Paolo (Grant 2015.0546 to F.B.) and CARIPLO Foundation Milano (Grant 20130879 to F.B.). The EU FP7 Integrating Project "Desire" (Grant no. 602531), to FB, FZ and AF is also acknowledged. D.A. was supported in 2018 by a Fondazione CARIGE fellowship (Genova).

\section{Compliance with ethical standards}

Conflict of interest The authors declare no conflict of interests.

Publisher's note: Springer Nature remains neutral with regard to jurisdictional claims in published maps and institutional affiliations.

\section{References}

1. Balestrini S, Milh M, Castiglioni C, Lüthy K, Finelli M, Taylor JC. TBC1D24 genotype - phenotype correlation Epilepsies and other neurologic features. Neurology. 2016;87:77-85.

2. Azaiez H, Booth KT, Bu F, Huygen P, Shibata SB, Shearer AE, et al. TBC1D24 mutation causes autosomal-dominant nonsyndromic hearing Loss. Hum Mutat. 2014;35:819-23.

3. Rehman AU, Santos-Cortez RLP, Morell RJ, Drummond MC, Ito $\mathrm{T}$, Lee $\mathrm{K}$, et al. Mutations in TBC1D24, a gene associated with epilepsy, also cause nonsyndromic deafness DFNB86. Am J Human Genet. 2014;94:144-52.

4. Zhang L, Hu L, Chai Y, Pang X, Yang T, Wu H. A Dominant mutation in the stereocilia-expressing gene TBC1D24 is a probable cause for nonsyndromic hearing impairment. Hum Mutat. 2014;35:814-8.

5. Finelli MJ, Oliver PL. TLDc proteins: new players in the oxidative stress response and neurological disease. Mamm Genome. 2017;28:395-406.

6. Fischer B, Lüthy K, Paesmans J, De Koninck C, Maes I, Swerts J, et al. Skywalker-TBC1D24 has a lipid-binding pocket mutated in epilepsy and required for synaptic function. Nat Struct Mol Biol. 2016;23:965-73.

7. Uytterhoeven V, Kuenen S, Kasprowicz J, Miskiewicz K, Verstreken P. Loss of Skywalker reveals synaptic endosomes as sorting stations for synaptic vesicle proteins. Cell. 2011;145:117-32.

8. Fernandes AC, Uytterhoeven V, Kuenen S, Wang YC, Slabbaert JR, Swerts J, et al. Reduced synaptic vesicle protein degradation at lysosomes curbs TBC1D24/sky-induced neurodegeneration. J Cell Biol. 2014;207:453-62.

9. Falace A, Filipello F, La Padula V, Vanni N, Madia F, De Pietri Tonelli D, et al. TBC1D24, an ARF6-interacting protein, is mutated in familial infantile myoclonic epilepsy. Am J Hum Genet. 2010;87:365-70.

10. Falace A, Buhler E, Fadda M, Watrin F, Lippiello P, PallesiPocachard E, et al. TBC1D24 regulates neuronal migration and 
maturation through modulation of the ARF6-dependent pathway. Proc Natl Acad Sci USA. 2014;111:2337-42.

11. Milh M, Falace A, Villeneuve N, Vanni N, Cacciagli P, Assereto $\mathrm{S}$, et al. Novel compound heterozygous mutations in TBC1D24 cause familial malignant migrating partial seizures of infancy. Hum Mutat. 2013;34:869-72.

12. Finelli MJ, Sanchez-Pulido L, Liu KX, Davies KE, Oliver PL. The evolutionarily conserved Tre2/Bub2/Cdc16 (TBC), lysin motif (LysM), domain catalytic (TLDc) domain is neuroprotective against oxidative stress. J Biol Chem. 2016;291:2751-63.

13. Barry J, Gu Y, Jukkola P, O’Neill B, Gu H, Mohler PJ, et al. Ankyrin-G directly binds to kinesin-1 to transport voltage-gated $\mathrm{Na}+$ channels into axons. Dev Cell. 2014;28:117-31.

14. McCormick DA, Shu Y, Yu Y. Neurophysiology: hodgkin and huxley model — still standing? Nature. 2007;445:E1-2.

15. Naundorf B, Wolf F, Volgushev M. Unique features of action potential initiation in cortical neurons. Nature. 2006;440:1060-3.

16. Namba T, Kibe Y, Funahashi Y, Nakamuta S, Takano T, Ueno T, et al. Pioneering axons regulate neuronal polarization in the developing cerebral cortex. Neuron. 2014;81:814-29.

17. Witteveen JS, Willemsen MH, Dombroski TC, van Bakel NH, Nillesen WM, van Hulten JA, et al. Haploinsufficiency of MeCP2interacting transcriptional co-repressor SIN3A causes mild intellectual disability by affecting the development of cortical integrity. Nat Genet. 2016;48:877-87.

18. Hernández-Deviez DJ, Roth MG, Casanova JE, Wilson JM. ARNO and ARF6 regulate axonal elongation and branching through downstream activation of phosphatidylinositol 4phosphate 5-kinase. Mol Biol Cell. 2004;15:111-20.

19. Eva R, Crisp S, Marland JRK, Norman JC, Kanamarlapudi V, ffrench-Constant $\mathrm{C}$, et al. ARF6 directs axon transport and traffic of integrins and regulates axon growth in adult DRG neurons. J Neurosci. 2012;32:10352-64.

20. Franssen EHP, Zhao R-R, Koseki H, Kanamarlapudi V, Hoogenraad CC, Eva R, et al. Exclusion of integrins from CNS axons is regulated by Arf6 activation and the AIS. J Neurosci. 2015;35:8359-75.

21. Vitriol EA, Zheng JQ. Growth cone travel in space and time: the cellular ensemble of cytoskeleton, adhesion, and membrane. Neuron. 2012;73:1068-81.

22. Sivadasan R, Hornburg D, Drepper C, Frank N, Jablonka S, Hansel A, et al. C9ORF72 interaction with cofilin modulates actin dynamics in motor neurons. Nat Neurosci. 2016;19:1610-8.

23. Bellani S, Mescola A, Ronzitti G, Tsushima H, Tilve S, Canale C, et al. GRP78 clustering at the cell surface of neurons transduces the action of exogenous alpha-synuclein. Cell Death Differ. 2014;21:1971-83.

24. Ageta-Ishihara N, Miyata T, Ohshima C, Watanabe M, Sato Y, Hamamura Y, et al. Septins promote dendrite and axon development by negatively regulating microtubule stability via HDAC6mediated deacetylation. Nat Commun. 2013;4:1-11.

25. Bonanomi D, Fornasiero EF, Valdez G, Halegoua S, Menegon A, Valtorta F. Identification of a developmentally regulated pathway of membrane retrieval in neuronal growth cones. J Cell Sci. 2009;121(Pt 22):3757-69.

26. Fruscione F, Valente P, Sterlini B, Romei A, Baldassari S, Fadda M, et al. PRRT2 controls neuronal excitability by negatively modulating $\mathrm{Na}+$ channel 1.2/1.6 activity. Brain. 2018;141:1000-16.

27. Campeau PM, Kasperaviciute D, Lu JT, Burrage LC, Kim C, Hori $\mathrm{M}$, et al. The genetic basis of DOORS syndrome: an exomesequencing study. Lancet Neurol. 2014;13:44-58.
28. Lozano R, Herman K, Rothfuss M, Rieger H, Bayrak-Toydemir P, Aprile D, et al. Clinical intrafamilial variability in lethal familial neonatal seizure disorder caused by TBC1D24 mutations. Am J Med Genet, Part A. 2016;170:3207-14.

29. Corbett MA, Bahlo M, Jolly L, Afawi Z, Gardner AE, Oliver KL, et al. A focal epilepsy and intellectual disability syndrome is due to a mutation in TBC1D24. Am J Hum Genet. 2010;87:371-5.

30. Yin J, Chen W, Chao ES, Soriano S, Wang L, Wang W, et al. Otud7a knockout mice recapitulate many neurological features of $15 \mathrm{q} 13.3$ microdeletion syndrome. Am J Human Genet. 2018;102:296-308.

31. Weckhuysen S, Mandelstam S, Suls A, Audenaert D, Deconinck T, Claes LRF, et al. KCNQ2 encephalopathy: Emerging phenotype of a neonatal epileptic encephalopathy. Ann Neurol. 2012;71:15-25.

32. Miceli F, Soldovieri MV, Ambrosino P, De Maria M, Migliore M, Migliore R, et al. Early-onset epileptic encephalopathy caused by gain-of-function mutations in the voltage sensor of Kv7.2 and Kv7.3 potassium channel subunits. J Neurosci. 2015;35:3782-93.

33. Devaux J, Abidi A, Roubertie A, Molinari F, Becq H, Lacoste C, et al. A Kv7.2 mutation associated with early onset epileptic encephalopathy with suppression-burst enhances Kv7/M channel activity. Epilepsia. 2016;57:e87-93.

34. Miura Y, Hongu T, Yamauchi Y, Funakoshi Y, Katagiri N, Ohbayashi N, et al. ACAP3 regulates neurite outgrowth through its GAP activity specific to Arf6 in mouse hippocampal neurons. Biochem J. 2016;473:2591-602.

35. Donaldson JG, Jackson CL. ARF family G proteins and their regulators: roles in membrane transport, development and disease. Nat Rev Mol Cell Biol. 2011;12:362-75.

36. Paleotti O, Macia E, Luton F, Klein S, Partisani M, Chardin P, et al. The small G-protein Arf6GTPrecruits the AP-2 adaptor complex to membranes. J Biol Chem. 2005;280:21661-6.

37. Kyung JW, Cho IH, Lee S, Song WK, Ryan TA, Hoppa MB, et al. Adaptor Protein 2 (AP-2) complex is essential for functional axogenesis in hippocampal neurons. Sci Rep. 2017;7:41620.

38. Krey JF, Dumont RA, Wilmarth PA, David LL, Johnson KR, Barr-Gillespie PG. ELMOD1 stimulates ARF6-GTP hydrolysis to stabilize apical structures in developing vestibular hair cells. J Neurosci. 2018;38:843-57.

39. Finelli MJ, Aprile D, Castroflorio E, Jeans A, Moschetta M, Chessum L, et al. The epilepsy-associated protein TBC1D24 is required for normal development, survival and vesicle trafficking in mammalian neurons. Hum Mol Genet. 2018;28:584-97.

40. Tona R, Chen W, Nakano Y, Reyes LD, Petralia RS, Wang YX, et al. The phenotypic landscape of a Tbc1d24 mutant mouse includes convulsive seizures resembling human early infantile epileptic encephalopathy. Hum Mol Genet. 2019. [Epub ahead of print].

41. Tagliatti E, Fadda M, Falace A, Benfenati F, Fassio A. Arf6 regulates the cycling and the readily releasable pool of synaptic vesicles at hippocampal synapse. eLife. 2016;5:1-18.

42. Pfaffl MW. A new mathematical model for relative quantification in real-time RT-PCR. Nucleic Acids Res. 2001;29:45e-45.

43. Valente P, Lignani G, Medrihan L, Bosco F, Contestabile A, Lippiello P, et al. Cell adhesion molecule L1 contributes to neuronal excitability regulating the function of voltage-gated $\mathrm{Na}+$ channels. J Cell Sci. 2016;129:1878-91.

44. Bean BP. The action potential in mammalian central neurons. Nat Rev Neurosci. 2007;8:451-65.

45. Shu Y, Duque A, Yu Y, Haider B, McCormick DA. Properties of action-potential initiation in neocortical pyramidal cells: evidence from whole cell axon recordings. J Neurophysiol. 2007;97:746-60. 